\title{
Ecological control strategy for cooperative autonomous vehicle in mixed traffic considering linear stability
}

\author{
Chaoru Lu \\ Department of Civil Engineering and Energy Technology, Oslo Metropolitan University, Oslo, Norway, and \\ Chenhui Liu \\ College of Civil Engineering, Hunan University, Changsha, China
}

\begin{abstract}
Purpose - This paper aims to present a cooperative adaptive cruise control, called stable smart driving model (SSDM), for connected and autonomous vehicles (CAVs) in mixed traffic streams with human-driven vehicles.

Design/methodology/approach - Considering the linear stability, SSDM is able to provide smooth deceleration and acceleration in the vehicle platoons with or without cut-in. Besides, the calibrated Virginia tech microscopic energy and emission model is applied in this study to investigate the impact of CAVs on the fuel consumption of the vehicle platoon and traffic flows. Under the cut-in condition, the SSDM outperforms ecological SDM and SDM in terms of stability considering different desired time headways. Moreover, single-lane vehicle dynamics are simulated for humandriven vehicles and CAVs.

Findings - The result shows that CAVs can reduce platoon-level fuel consumption. SSDM can save the platoon-level fuel consumption up to 15\%, outperforming other existing control strategies. Considering the single-lane highway with merging, the higher market penetration of SSDMequipped CAVs leads to less fuel consumption.

Originality/value - The proposed rule-based control method considered linear stability to generate smoother deceleration and acceleration curves. The research results can help to develop environmental-friendly control strategies and lay the foundation for the new methods.
\end{abstract}

Keywords Adaptive cruise control, Connected and autonomous vehicle (CAV), Linear stability

Paper type Research paper

\section{Introduction}

In recent years, autonomous driving technologies started to be implemented to provide transport services. Since 2019, Level 3 autonomous vehicles have been tested in the Oslo area to provide public transport services. Until December 2020, more than 29,000 passengers have been served and over 33,000 kilometers have been operated by autonomous buses [1]. Moreover, the number of vehicles equipped with adaptive cruise control (ACC) in the transport system is expected to increase. Accordingly, autonomous driving-related topics attracted the attention of researchers all over the world. As one of the core parts of the autonomous vehicle, a proper control strategy will significantly improve the performance of autonomous vehicles and/or transport systems in terms of traffic efficiency, safety and fuel consumption (Kamal et al., 2011; Kesting et al., 2008; Li et al., 2015a; Luo et al., 2015; Mahdinia et al., 2020; Yuan et al., 2009).

Existing control strategies for autonomous vehicles can be divided into three major categories: optimization, machine

The current issue and full text archive of this journal is available on Emerald Insight at: https://www.emerald.com/insight/2399-9802.htm

Journal of Intelligent and Connected Vehicles $4 / 3(2021) 115-124$

Emerald Publishing Limited [ISSN 2399-9802] [DOI 10.1108/JICV-08-2021-0012] learning and physical models. Desjardins and Chaib-Draa (2011) proposed a reinforcement-learning-based longitudinal following strategy that applied function approximation techniques and gradient-descent learning algorithms to modify the following policy. Based on a machine-learning method to detect special maneuvers and predict trajectory, Kazemi et al. (2018) develop a stochastic model predictive controller to guarantee safety. Li and Görges (2020) developed an ecological ACC by using reinforcement learning with a novel actor-gearcritic architecture. Recently, Lin et al. (2021) compared the ACC methods based on deep learning and model predictive

\footnotetext{
(C) Chaoru Lu and Chenhui Liu. Published in Fournal of Intelligent and Connected Vehicles. Published by Emerald Publishing Limited. This article is published under the Creative Commons Attribution (CC BY 4.0) licence. Anyone may reproduce, distribute, translate and create derivative works of this article (for both commercial and non-commercial purposes), subject to full attribution to the original publication and authors. The full terms of this licence maybe seen at http://creativecommons.org/licences/ by/4.0/legalcode
}

The research is part of the project China-Norway Partnership in Smart Sustainable Metropolitan Transport (COMet) (UTF-2020/10115), funded by the Norwegian Agency for International Cooperation and Quality Enhancement in Higher Education (Diku). Any opinions, findings, and conclusions expressed in this material are those of the authors and do not necessarily reflect the views of these organizations.

Received 14 August 2021

Revised 15 October 2021

Accepted 19 October 2021 
control. They pointed out that the deep learning method can train a policy very close to the optimal. However, the deep learning method has difficulties to deal with the case that fall outside the training dataset. Recently, Kuutti et al. (2021) conducted a comprehensive review on deep learning-based control strategies. They indicated the drawbacks of these strategies in computation, architecture selection, adaptability, generalization, etc. Moreover, the explainability of the deep learning-based method is also a challenge (Fazi, 2020). Another widely applied approach to develop autonomous vehicle control strategies is the optimization method. Sakhdari and Azad (2018) proposed an adaptive tube-based nonlinear model predictive control method to control autonomous vehicles and reduce the energy cost of plug-in hybrid electric vehicles. Considering the predicted traffic state, the ecological driving system proposed by Huang et al. (2018) could optimize the travel speed profile to reduce fuel consumption. Considering the fallback procedure, Xue et al. (2019) proposed an adaptive model predictive control strategy to avoid potential collisions. To improve ride comfort and safety, He et al. (2020) proposed a stair-like predictive cruise control that can significantly reduce the computational cost. Li and Görges (2020) proposed an optimal control strategy by using reinforcement learning with a novel actor-gear-critic architecture to reduce fuel consumption while ensuring the safe inter-vehicle distance. Wang et al. (2020) proposed the idea of dynamically optimizing the information flow topologies for cooperative ACC and enhance its performance in terms of string stability. Tajeddin et al. (2020) proposed a control strategy to provide optimal speed and lane-to-drive in realtime. The optimization objective includes safety, energy efficiency and desired speed tracking. Based on the hardwarein-loop experiments, the result shows that the proposed method can reduce up to $27 \%$ of energy consumption compared to human drivers. Moreover, Nunen et al. (2019) designed a model predictive control strategy to guarantee string stability. Zhu et al. (2020) proposed a linear matrix inequalitiesbased method to synthesize string-stable control strategy with low computational cost. The results from numerical experiments validated the performance of these proposed methods.

Moreover, numerous ACCs are developed based on physical models. To simulate human-driven vehicles, several carfollowing models are introduced in the past decades (Anesiadou et al., 2021; Bando et al., 1995; Gipps, 1981; Jiang et al., 2001; Lenz et al., 1999; Newell, 1961; Tampere, 2004). Based on existing car-following models, Davis (2004) and Kesting et al. (2010) developed several ACC strategies to investigate the impact of autonomous vehicles on traffic stability and capacity. Recently, Yang et al. (2020) developed a novel hierarchical ecological cooperative adaptive cruise control based on combined feedforward-feedback control. The results showed that the proposed model can maintain a stable platoon and reduce fuel consumption. Huang et al. (2020) proposed a CACC strategy considering the time-varying lags function. The linear stability has been investigated based on Lyapunov function theory. Moreover, the proposed CACC can significantly reduce fuel consumption compared to humandriven vehicles.

In recent years, researchers started to adopt field tests and explore the performance of autonomous control strategies.
Gunter et al. (2019, 2020) conducted a set of car-following experiments to collect field data from ACC-equipped vehicles. They evaluated calibrated models and pointed out that the models are string unstable. Similarly, Makridis et al. (2020) conduct a field experiment with five ACC-equipped vehicles to explore the properties of ACC systems and their performances under real-world situations. The results showed that the ACC system used in the experiment leads to instability of the carplatoon even for slight perturbations. Recently, Shang and Stern (2021) investigated the traffic impact of commercially available ACC vehicles, which is based on intelligent driver model. The result shows that commercially available ACC vehicles may reduce highway bottleneck capacity up to $35 \%$ because of string unstable. Meanwhile, they also indicated that the string stability and time headway are major impact factors, leading to bottleneck capacity reduction caused by the commercially available ACC vehicles.

In summary, several ACC/CACC systems have been developed to control connected and autonomous vehicle (CAVs). Most of the ecological control strategies are formulated as optimization problems, such as particle swarm optimization and model predictive control. Because optimization-based approaches are complicated and computation-intensive, rule-based control strategies have monopolized the production vehicle market because of their low computational demand, natural adaptability to online applications and reliability (Enang and Bannister, 2017; Lu et al., 2019a, 2019b). However, to the best of the authors' knowledge, none of the existing control strategies considered fuel efficiency or linear stability at the same time. To bridge the existing gap, a rule-based autonomous vehicle control strategy is proposed.

The rest of the paper is organized as follows. Section 2 presents the rule-based control strategy that considers fuel efficiency and linear stability. Section 3 evaluates the performance of the proposed control method by simulating different traffic situations. The conclusion is presented in Section 4 .

\section{Methodology}

Recently, a rule-based ACC, named smart driver model (SDM), is proposed to address the instability of IDM under homogenous traffic conditions ( $\mathrm{Lu}$ and Aakre, 2018). The acceleration profile of the following vehicle equipped with $\mathrm{SDM}$ is determined by the following equation:

$$
a_{S D M}^{n}=a_{\max }\left[1-\left(\frac{v_{n}}{v_{0}}\right)^{4}\right]-\frac{a_{\max }\left[1-\left(\frac{v_{n}}{v_{0}}\right)^{4}\right]+\frac{v_{n}^{2}-v_{n-1}^{2}}{2 x}}{\exp \left(\frac{x}{s_{0}+v_{n} \times T}-1\right)}
$$

$$
\begin{aligned}
& \text { where, } \\
& \begin{aligned}
a_{S D M}^{n}= & \text { acceleration of the following vehicle that is equipped } \\
& \text { with } \operatorname{SDM}\left(\mathrm{m} / \mathrm{s}^{2}\right) ; s_{0} \text { is the standstill distance between } \\
& \text { stopped vehicles }(\mathrm{m}) ; a_{\max } \text { is the maximum } \\
& \text { acceleration }\left(\mathrm{m} / \mathrm{s}^{2}\right) ;
\end{aligned} \\
& \begin{aligned}
\Delta x \quad & \text { spacing between the leading and the following vehicle } \\
& (\mathrm{m}) ;
\end{aligned} \\
& T=\text { desired time headway }(s) ;
\end{aligned}
$$


$v_{0} \quad=$ desired speed $(\mathrm{m} / \mathrm{s})$

$v_{n} \quad=$ speed of the following vehicle $(\mathrm{m} / \mathrm{s}) ;$ and $v_{n-1}$

$=$ speed of the leading vehicle $(\mathrm{m} / \mathrm{s})$.

Considering the synchronized flow and congestion condition, where the driving behavior of the following vehicle is impacted by the leading vehicle, the impact of speed control terms in equation (1), $\left.11-\left(\frac{v_{n}}{v_{0}}\right)^{4}\right]$, can be ignored. Therefore, equation (1) can be simplified as follows:

$$
a_{S D M}^{n}=a_{\max }-\frac{a_{\max }+\frac{v_{n}^{2}-v_{n-1}^{2}}{2 x}}{\exp \left(\frac{x}{s_{0}+v_{n} \times T}-1\right)}
$$

Based on the SDM (Lu and Aakre, 2018) and ecological SDM (EcoSDM) (Lu et al., 2019a, 2019b), a rule-based Ecological CACC, named stable SDM (SSDM), is proposed considering the platoon-level string stability. With regard to connected vehicles and autonomous vehicles, we assume that connected vehicles are capable of communicating with other connected vehicles through vehicle-to-vehicle communication (Davis, 2017). Moreover, the onboard sensors measure vehicle speed, space headway and relative speed with respect to the preceding vehicle on regular time intervals (Wang et al., 2018).

By introducing the stabilization parameter into SDM [equation (2)], the acceleration profile of the following vehicle equipped with the proposed SSDM is determined by the following equation:

$$
a_{S S D M}^{n}=a_{\max }-\frac{a_{\max }+\frac{v_{n}^{2}-v_{n-1}^{2}}{2 x}}{\exp \left(\frac{x}{s_{0}+v_{n} \times T}-1-\beta \times \frac{v_{n}}{v_{0}}\right)}
$$

where,

$$
\begin{aligned}
a_{S S D M}^{n}= & \text { acceleration of the following vehicle that is equipped } \\
& \text { with SSDM }\left(\mathrm{m} / \mathrm{s}^{2}\right) ; \text { and } \\
\beta \quad= & \text { stabilization parameter. }
\end{aligned}
$$

According to the existing string stability analysis studies (Chen et al., 2013; Pei et al., 2016; Wilson and Ward, 2011), the general form of time-continuous car-following models is formulated as follows:

$$
\ddot{x}_{n}=f\left(v_{n}(t-\tau), s_{n}(t-\tau), v_{n}(t-\tau)\right)
$$

where,

$f \quad=$ a general nonlinear function;

$\ddot{x}_{n} \quad=$ acceleration of the following vehicle in the platoon; $s_{\mathrm{n}}$ is the space headway, $s_{\mathrm{n}}=s_{0}+v_{\mathrm{n}} \times T ; \tau$ is the delay; and

$\Delta v_{\mathrm{n}}=$ relative velocity between the leading and following vehicles, $\Delta v_{\mathrm{n}}=v_{n-1}-v_{\mathrm{n}}$.

In this study, a platoon mixed with SSDM-equipped vehicles and human-driven vehicles is considered. To achieve the platoon-level string stability, the parameter $(\beta)$ of SSDM is determined, based on the string stability condition proposed by Talebpour and Mahmassani (2016) and Sun et al. (2018), as follows:

$$
\begin{aligned}
& (1-\varphi)\left[\frac{f_{v}^{o 2}}{2}-f_{v}^{o} f_{v}^{o}-f_{s}^{o}+\tau_{1} f_{v}^{o} f_{s}^{o}\right]\left[f_{s}^{S}\right]^{2} \\
& +\varphi\left[\frac{f_{v}^{S 2}}{2}-f_{v}^{S} f_{v}^{S}-f_{s}^{S}+\tau_{2} f_{v}^{S} f_{s}^{S}\right]\left[f_{s}^{O}\right]^{2}>0
\end{aligned}
$$

Where, $f_{v}^{O}=\left.\frac{\partial f}{\partial v_{n}}\right|_{\left(v_{e}, s_{e}, 0\right)}, \quad f_{v}^{O}=\left.\frac{\partial f}{\partial v_{n}}\right|_{\left(v_{e}, s_{e}, 0\right)}$ and $f_{s}^{O}=\left.\frac{\partial f}{\partial s_{n}}\right|_{\left(v_{e}, s_{e}, 0\right)}$ are partial derivatives of human-driven model, which represent the human-driven vehicles in the platoon; $f_{v}^{S}=\left.\frac{\partial f}{\partial v_{n}}\right|_{\left(v_{e}, s_{e}, 0\right)}$, $f_{v}^{S}=\left.\frac{\partial f}{\partial v_{n}}\right|_{\left(v_{e}, s_{e}, 0\right)}$ and $f_{s}^{S}=\left.\frac{\partial f}{\partial s_{n}}\right|_{\left(v_{e}, s_{e}, 0\right)}$ are partial derivatives of SSDM model, which represent the autonomous vehicles in the platoon; and $\varphi$ denotes the penetration rate of autonomous vehicles in the platoon, $\varphi=\frac{M}{N}$.

By substituting equations (6)-(9) into the stability condition [equation (4)], we have the following:

$$
\begin{gathered}
f_{[\text {jmath }] v}^{S}=\frac{v_{e}}{s_{e} \times \exp \left(-\beta \times \frac{v_{n}}{v_{0}}\right)} \\
f_{s}^{S}=-\frac{a_{\max }}{s_{e} \times \exp \left(-\beta \times \frac{v_{n}}{v_{0}}\right)} \\
f_{v}^{S}=\frac{\left(\frac{\beta}{v_{0}}-\frac{T}{s_{e}}\right) \times a_{\max }}{\exp \left(-\beta \times \frac{v_{n}}{v_{0}}\right)} \\
(1-\varphi)\left[\frac{f_{v}^{O 2}}{2}-f_{v}^{O} f_{v}^{O}-f_{s}^{O}+\tau_{1} f_{v}^{o} f_{s}^{o}\right]\left[\frac{1}{s_{e}}\right]^{2} \\
+\varphi\left[\frac{\left(\frac{\beta}{v_{0}}-\frac{T}{s_{e}}\right)^{2}}{2}-\frac{\left(\frac{\beta}{v_{0}}-\frac{T}{s_{e}}\right) \times v_{e}}{a_{\max } \times s_{e}}+\frac{\exp \left(-\beta \times \frac{v_{e}}{v_{0}}\right)}{a_{\max } \times s_{e}}\right. \\
\left.-\tau_{2} \frac{\left(\frac{\beta}{v_{0}}-\frac{T}{s_{e}}\right)}{s_{e}}\right]\left[f_{s}^{O}\right]^{2}>0
\end{gathered}
$$

Because $\exp \left(-\beta \times \frac{v_{e}}{v_{0}}\right)>0$, we have:

$$
\begin{aligned}
& \frac{\left(\frac{\beta}{v_{0}}-\frac{T}{s_{e}}\right)^{2}}{2}-\frac{\left(\frac{\beta}{v_{0}}-\frac{T}{s_{e}}\right) \times v_{e}}{a_{\max } \times s_{e}}+\frac{\exp \left(-\beta \times \frac{v_{e}}{v_{0}}\right)}{a_{\max } \times s_{e}}-\tau_{2} \frac{\left(\frac{\beta}{v_{0}}-\frac{T}{s_{e}}\right)}{s_{e}} \\
& >\frac{\left(\frac{\beta}{v_{0}}-\frac{T}{s_{e}}\right)^{2}}{2}-\frac{\left(\frac{\beta}{v_{0}}-\frac{T}{s_{e}}\right) \times v_{e}}{a_{\max } \times s_{e}}-\tau_{2} \frac{\left(\frac{\beta}{v_{0}}-\frac{T}{s_{e}}\right)}{s_{e}}
\end{aligned}
$$

As a result, the stability condition is derived as follows:

$$
\begin{aligned}
& \frac{\left(\frac{\beta}{v_{0}}-\frac{T}{s_{e}}\right)^{2}}{2}-\frac{\left(\frac{\beta}{v_{0}}-\frac{T}{s_{e}}\right) \times v_{e}}{a_{\max } \times s_{e}}-\tau_{2} \frac{\left(\frac{\beta}{v_{0}}-\frac{T}{s_{e}}\right)}{s_{e}}>A \\
& A=\frac{(M-N)}{M}\left[\frac{f_{v}^{O 2}}{2}-f_{v}^{O} f_{v}^{O}-f_{s}^{O}+\tau_{1} f_{v}^{o} f_{s}^{o}\right]\left[\frac{1}{f_{s}^{O} \times s_{e}}\right]^{2}
\end{aligned}
$$

By solving the stability condition [equation (12)], we have the following:

$$
\beta>v_{0}\left[\sqrt{2 A+\left(\frac{v_{e}+a_{\max } \times \tau_{2}}{a_{\max } \times s_{0}+v_{e} T}\right)^{2}}+\frac{v_{e}+\left(\tau_{2}+T\right) \times a_{\max }}{a_{\max } \times s_{0}+v_{e} T}\right]
$$

Therefore, the stabilization parameter in this study is as follows: 


$$
\beta=v_{0}\left[\sqrt{2 A+\left(\frac{v_{e}+a_{\max } \times \tau_{2}}{a_{\max } \times s_{0}+v_{e} T}\right)^{2}}+\frac{v_{e}+\left(\tau_{2}+T\right) \times a_{\max }}{a_{\max } \times s_{0}+v_{e} T}\right]
$$

Because the following vehicle is targeting at the speed of the leading vehicle, the speed of the leading vehicle is set as the equilibrium speed. The stabilization parameter is calculated as follows:

$$
\beta=\left\{\begin{array}{c}
v_{0}\left[\sqrt{2 A+\left(\frac{v_{n-1}+a_{\max } \times \tau_{2}}{a_{\max } \times\left(s_{0}+v_{n-1} \times T\right)}\right)^{2}}+\frac{v_{n-1}+\left(\tau_{2}+T\right) \times a_{\max }}{a_{\max } \times\left(s_{0}+v_{n-1} \times T\right)}\right], A>0 \\
v_{0}\left[\frac{2 v_{n-1}+\left(2 \tau_{2}+T\right) \times a_{\max }}{a_{\max } \times\left(s_{0}+v_{n-1} \times T\right)}\right], \quad A \leq 0
\end{array}\right.
$$

The environmental benefit may vary with the location in the string of mixed traffic (Ioannou and Stefanovic, 2005). According to the study conducted by Ioannou and Stefanovic (2005), the CAV located in the front of the platoon must be able to attenuate most of the disturbance from the humandriven vehicle in front of it to achieve better stabilization and ecological effects. Therefore, we weighted the stabilization parameter based on the position of CAVs in the platoon. The SSDM is formulated as follows:

$$
a_{S S D M}^{n}=a_{\max }-\frac{a_{\max }+\frac{v_{n}^{2}-v_{n-1}^{2}}{2 x}}{\exp \left(\frac{x}{s_{0}+v_{n} \times T}-1-\beta \times \frac{v_{n}}{v_{0}}\right)}
$$

Especially, when the delays are ignored, equation (16) is simplified as follows:

$$
=\left\{\begin{array}{c}
\beta_{\text {stable }}\left[\sqrt{2 A+\left(\frac{v_{n-1}}{a_{\max } \times\left(s_{0}+v_{n-1} \times T\right)}\right)^{2}}+\frac{v_{n-1}+T \times a_{\max }}{a_{\max } \times\left(s_{0}+v_{n-1} \times T\right)}\right], A>0 \\
v_{0}\left[\frac{2 v_{n-1}+T \times a_{\max }}{a_{\max } \times\left(s_{0}+v_{n-1} \times T\right)}\right], \quad A \leq 0
\end{array}\right.
$$

Same as in the study conducted by Talebpour and Mahmassani (2016), the IDM (Kesting et al., 2010) is used in this paper to simulate the human-driven connected vehicles. The IDM is formulated as follows:

$$
\begin{gathered}
a_{I D M}^{n}=a_{\max }\left[1-\left(\frac{v_{n}}{v_{0}}\right)^{4}-\left(\frac{s^{*}}{x}\right)^{2}\right] \\
s^{*}=s_{0}+v_{n} T+\frac{v_{n}\left(v_{n}-v_{n-1}\right)}{2 \sqrt{a_{\max } b}}
\end{gathered}
$$

where,

$$
\begin{aligned}
a_{I D M}^{n}= & \text { acceleration of the following vehicle based on the } \\
& \operatorname{IDM~}\left(\mathrm{m} / \mathrm{s}^{2}\right) ; s^{*} \text { is the desired headway space }(\mathrm{m}) ; \\
& \text { and } \\
b= & \text { desired deceleration }\left(\mathrm{m} / \mathrm{s}^{2}\right) .
\end{aligned}
$$

According to the study conducted by $\mathrm{Li}$ et al. (2015b), the partial derivatives of IDM at equilibrium can be calculated as follows:

$$
\begin{array}{r}
f_{v}^{O}=-\frac{v_{e}}{s_{0}+v_{e} T} \sqrt{\frac{a_{\max }}{b}} \\
f_{s}^{O}=\frac{2 a_{\max }}{s_{0}+v_{e} T} \\
f_{v}^{O}=-\frac{4 a_{\max } v_{e}^{3}}{v_{0}^{4}}-\frac{2 a_{\max } T}{s_{0}+v_{e} T}
\end{array}
$$

By substituting equations (21)-(23) into the stability condition [equation (13)], we have the following:

$$
\begin{aligned}
A= & \frac{(M-N)}{M}\left[\frac{\left(\frac{4 a v_{e}^{3}}{v_{0}^{4}}+\frac{2 a_{\max } T}{s_{0}+v_{e} T}\right)^{2}}{2}-\left(\frac{v_{e}}{s_{0}+v_{e} T} \sqrt{\frac{a_{\max }}{b}}\right)\right. \\
& \left(\frac{4 a_{\max } v_{e}^{3}}{v_{0}^{4}}+\frac{2 a_{\max } T}{s_{0}+v_{e} T}\right)-\frac{2 a_{\max }}{s_{0}+v_{e} T}-\tau_{1} \frac{2 a_{\max }}{s_{0}+v_{e} T} \\
& \left.\left(\frac{4 a_{\max } v_{e}^{3}}{v_{0}^{4}}+\frac{2 a_{\max } T}{s_{0}+v_{e} T}\right)\right]\left[\frac{1}{2 a_{\max }}\right]^{2}
\end{aligned}
$$

\section{Numerical simulations}

Numerical simulation has been widely used to compare techniques in transportation research to evaluate performance (Zheng, 2021). In this section, numerical experiments are conducted to evaluate the performance of SSDM-equipped vehicles by comparing them with existing control strategies. The parameters of ACC or car-following models are based on the parameters used by existing studies (Chen et al., 2009; Kesting et al., 2010; Lu and Aakre, 2018), where the maximum acceleration is $1.4 \mathrm{~m} / \mathrm{s}^{2}$; the desired time headway is $1.6 \mathrm{~s}$; and the standstill distance is $1.5 \mathrm{~m}$. Based on the work conducted by Li et al. (2015b), an open-boundary single-lane highway with $30 \mathrm{~m} / \mathrm{s}$ speed limit is simulated considering different scenarios. The speed and distance between the leading and following vehicles are measured by the sensor at each time step.

\subsection{Vehicle platoon with cut-in}

First of all, the performance of SSDM is compared with SDM and EcoSDM by simulating the cut-in scenario, which has been widely applied in existing studies (Davis, 2007; Lu and Aakre, 2018; Milanés and Shladover, 2016). As shown in Figure 1, the oscillations from the cut-in vehicle are reduced by the 4 th to the 14 th vehicles equipped with control models when the desired time headway is $1.5 \mathrm{~s}$. When the desired time headway is $1 \mathrm{~s}$, the SDM is amplifying the oscillation while EcoSDM and SSDM are stable. Moreover, the SSDM outperforms EcoSDM and SIDM in terms of stability considering different desired time headways. The reason is that SSDM considered platoonlevel stability.

\subsection{Vehicle platoon without cut-in}

Driving cycles, which represent different driving scenarios, have been widely applied in simulations to evaluate the performance of control strategies (Li and Görges, 2020; Lu et al., 2019a, 2019b; Lin et al., 2021). In this study, a vehicle platoon with 100 vehicles is simulated on a single-lane road where the leading vehicle follows the driving cycle of federal test procedure (FTP) [2]. In this section, the CAVs are equipped with different control strategies, i.e. SDM, EcoSDM and SSDM. Therefore, the stability and safety performance of SSDM has been explored by comparing with SDM and EcoSDM.

As shown in Figure 2, SSDM is able to stabilize the string faster than SDM and EcoSDM. Moreover, SSDM provides smoother deceleration and acceleration than SDM and 
Figure 1 Acceleration profiles of vehicles equipped with SDM, EcoSDM and SSDM under cut-in situation considering different desired time headways

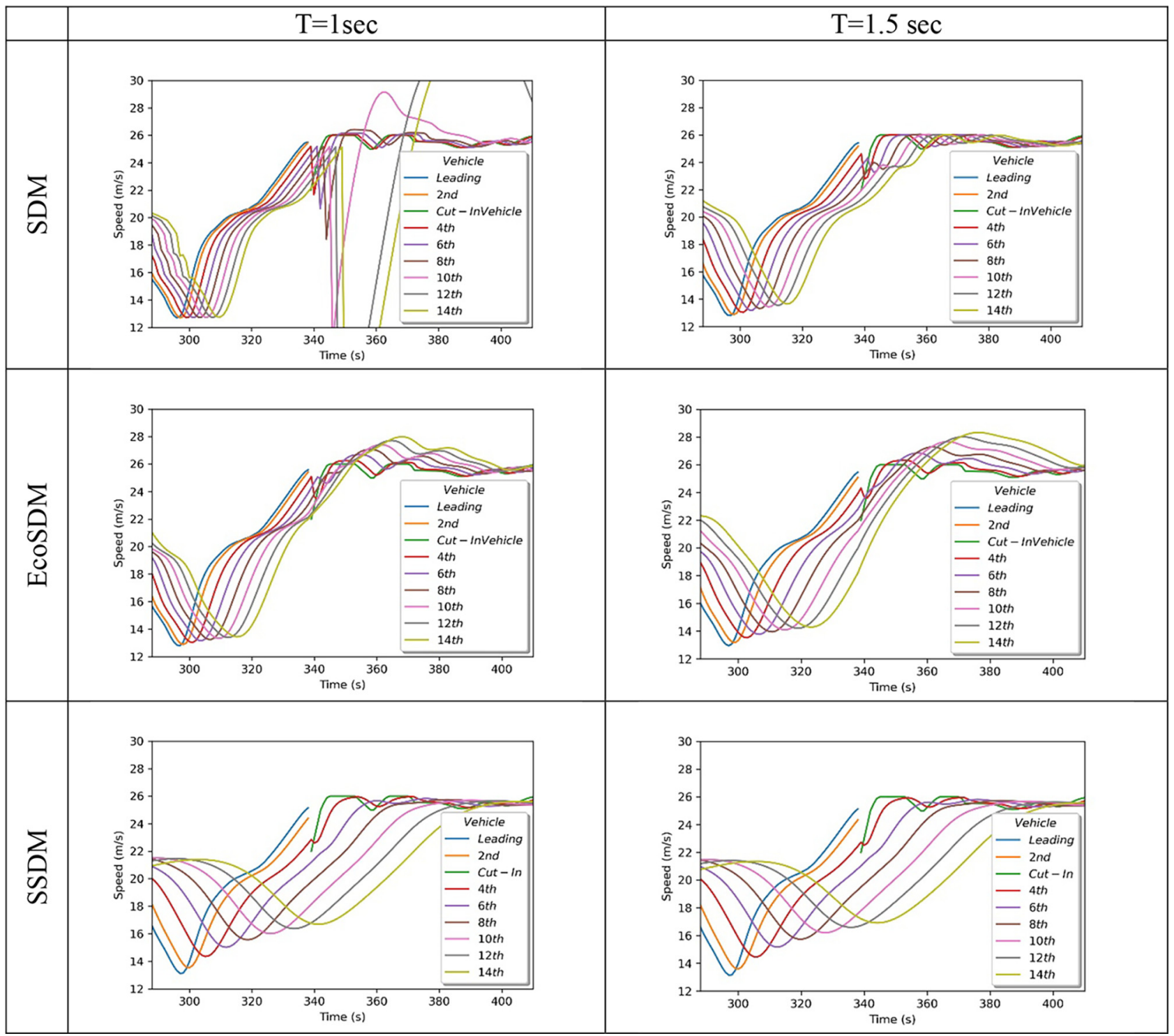

EcoSDM. Therefore, it requires fewer CAVs in the platoon to maintain a stable string if CAVs are equipped with SSDM.

To investigate the safety performance of SSDM, the collision sensitivity coefficient (CSC) by Jiao et al. (2021) is used in this study. The CSC is calculated as follows:

$$
C S C=\frac{v_{n-1}(t)-v_{n}(t)}{x(t)-l_{c}}
$$

If CSC is less than 0 , the probability of a collision between vehicles increases. Alternatively, if CSC is larger or equal to 0, the probability of a collision between vehicles is small.

As shown in Figure 3, SSDM leads to a safer platoon than SDM and EcoSDM. Together with the aforementioned result, the control strategies with better stabilization ability will lead to the better platoon-level safety performance.

Moreover, a platoon with 20 vehicles is simulated to evaluate the fuel consumption of different control strategies. In this study, IDM is used to model human-driven vehicles and used as the baseline. The Virginia tech microscopic energy and emission (VT-Micro) model (Ahn et al., 2002; Rakha and Ahn, 2004), which is calibrated by $\mathrm{Lu}$ et al. (2018), is used to calculate the fuel consumption of vehicles. Three driving cycles, which are FTP, NY City cycle (NYCC) and LA92 dynamometer driving schedule (LA92DDS) [3], are considered in this study. The performance of different control strategies is shown in Table 1. Considering FTP, NYCC and LA92DDS, three control strategies have similar average speed and average acceleration, which means that the travel times of vehicles equipped with these control strategies are similar. Overall, SSDM has the smoothest speed profile with the smallest acceleration variance. Figure 4 shows the fuel consumption enhancement of SDM, EcoSDM and SSDM considering different driving cycles. The result shows that SSDM outperforms EcoSDM and SDM in terms of fuel consumption considering FTP, NYCC and LA92DDS. Moreover, SSDM can reduce the platoon-level fuel consumption by up to $15 \%$. 
Figure 2 Acceleration profiles of vehicle platoon equipped with SDM, ECOSDM and SSDM

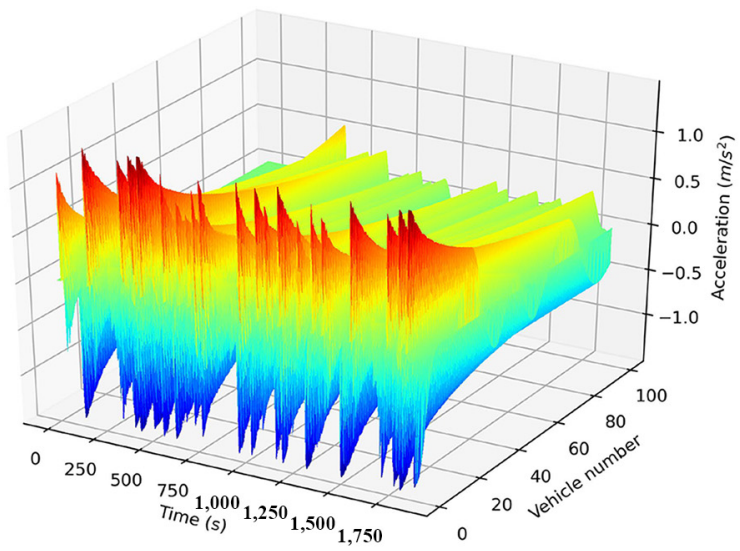

(a)

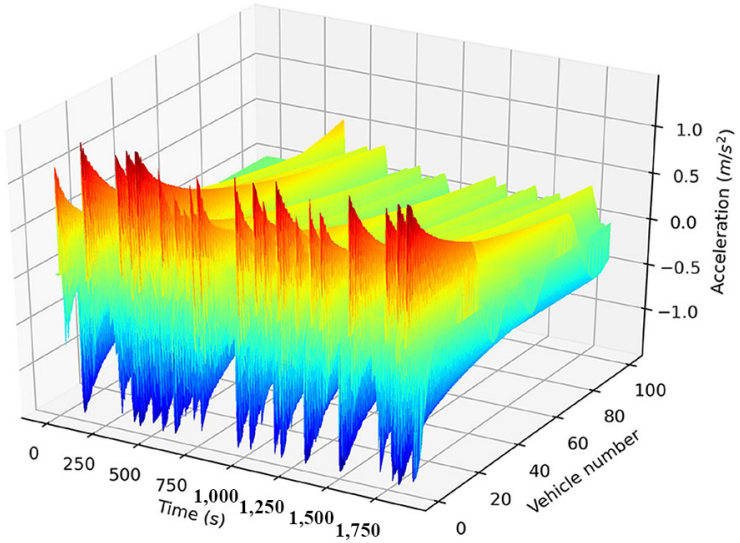

(b)

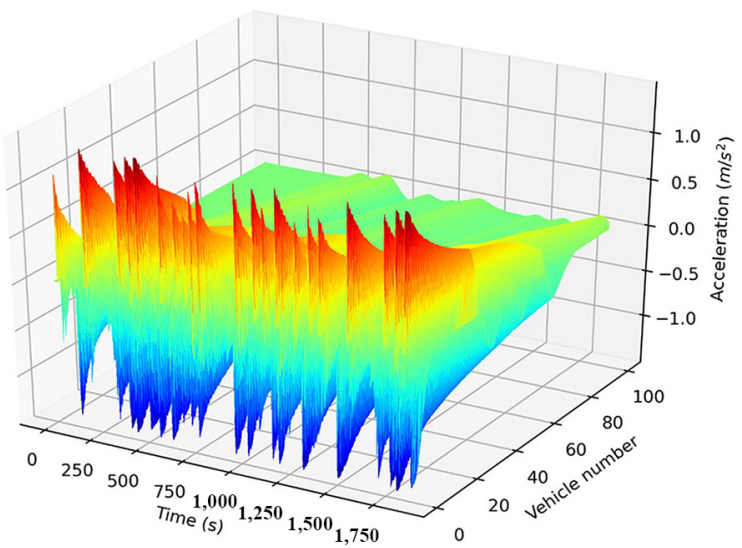

(c)

Note: Acceleration profiles of vehicle platoon equipped with SDM, EcoSDM, and SSDM

\subsection{Merging}

In this section, a hypothetical single-lane highway with a single-lane on-ramp (Figure 5) is applied to evaluate the performance of the proposed control strategy. The merging maneuver proposed by Davis (2007) is used in this study. Because the proposed control
Figure 3 CSC profiles of vehicle platoon equipped with SDM, EcoSDM and SSDM

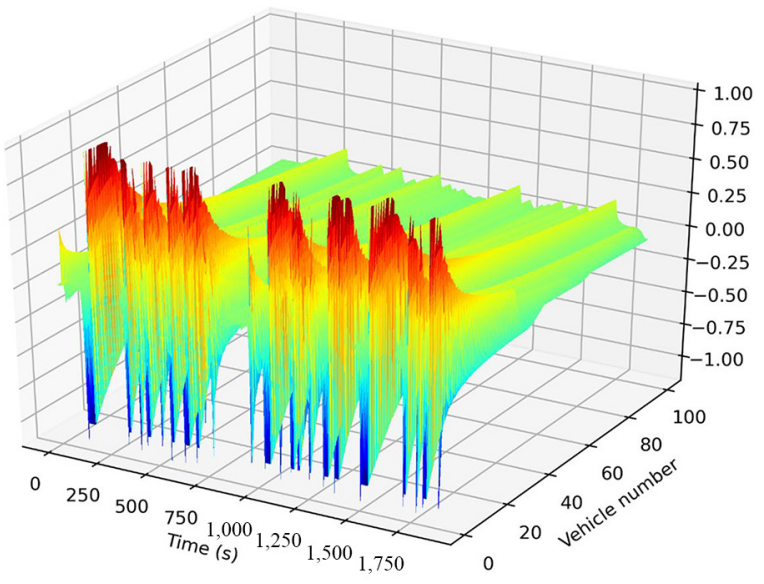

(a)

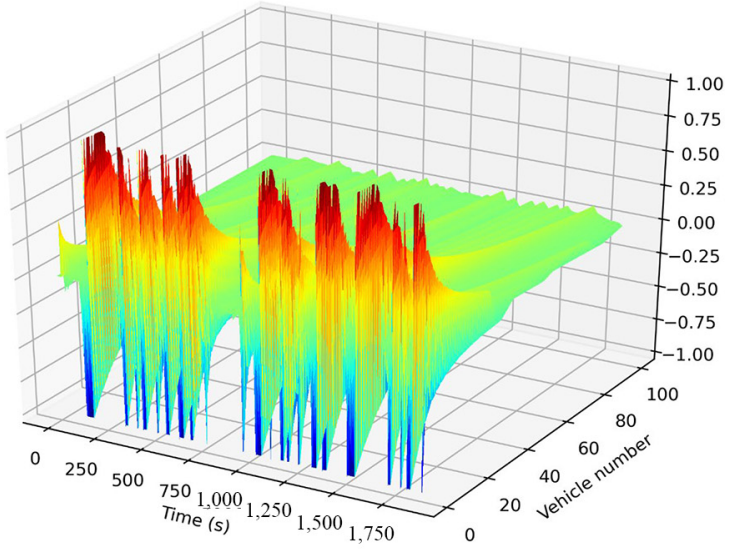

(b)

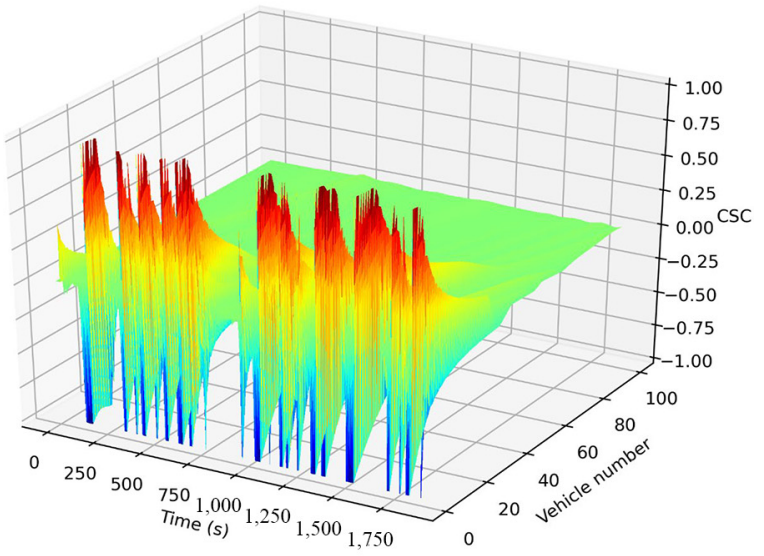

(c)

Note: CSC profiles of vehicle platoon equipped with SDM, EcoSDM, and SSDM

strategy is able to consider string stability, a congested traffic status is considered in this study. Therefore, the main lane and ramp flow are set to $2000 \mathrm{veh} / \mathrm{h}$ and $600 \mathrm{veh} / \mathrm{h}$, respectively. As shown in Figure 6, the higher market penetration of SSDM-equipped CAVs leads to less fuel consumption. 
Table 1 Performance of different control strategies

\begin{tabular}{|c|c|c|c|c|c|}
\hline Driving cycle & Characteristic & Statistics & SDM & EcoSDM & SSDM \\
\hline \multirow[t]{4}{*}{ FTP } & Speed $(\mathrm{m} / \mathrm{s})$ & Average & 9.39 & 9.38 & 9.37 \\
\hline & & Standard deviation & 7.05 & 6.85 & 6.8 \\
\hline & Acceleration $\left(\mathrm{m} / \mathrm{s}^{2}\right)$ & Average & 0.007 & 0.007 & 0.007 \\
\hline & & Standard deviation & 0.501 & 0.458 & 0.446 \\
\hline \multirow[t]{4}{*}{ NYCC } & Speed (m/s) & Average & 3.2 & 3.2 & 3.2 \\
\hline & & Standard deviation & 3.27 & 3.13 & 3.04 \\
\hline & Acceleration $\left(\mathrm{m} / \mathrm{s}^{2}\right)$ & Average & 0.001 & 0.001 & 0.001 \\
\hline & & Standard deviation & 0.398 & 0.361 & 0.331 \\
\hline \multirow[t]{4}{*}{ LA992DDS } & Speed (m/s) & Average & 10.98 & 10.98 & 10.98 \\
\hline & & Standard deviation & 8.74 & 8.51 & 8.46 \\
\hline & Acceleration $\left(\mathrm{m} / \mathrm{s}^{2}\right)$ & Average & 0.002 & 0.002 & 0.002 \\
\hline & & Standard deviation & 0.57 & 0.519 & 0.499 \\
\hline
\end{tabular}

Figure 4 Platoon-level fuel saving of CAVs with different driving cycles

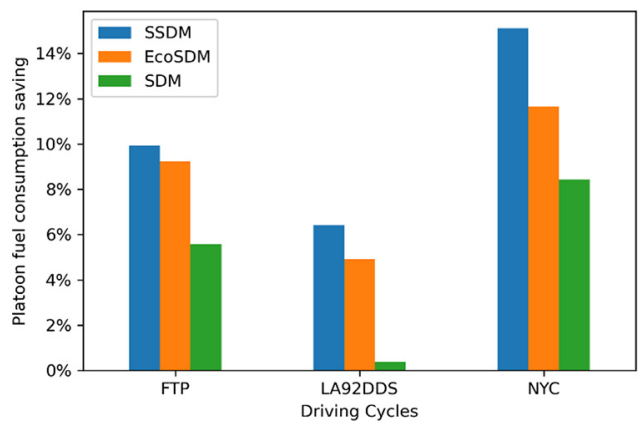

Figure 5 Scenario of merging simulation

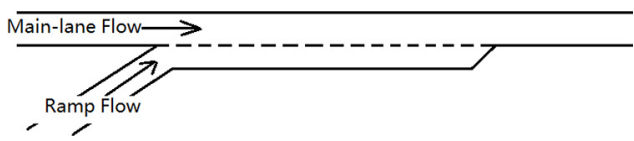

Figure 6 Cumulative fuel consumption on the simulated freeway segment considering different market penetration of SSDM-equipped CAVs

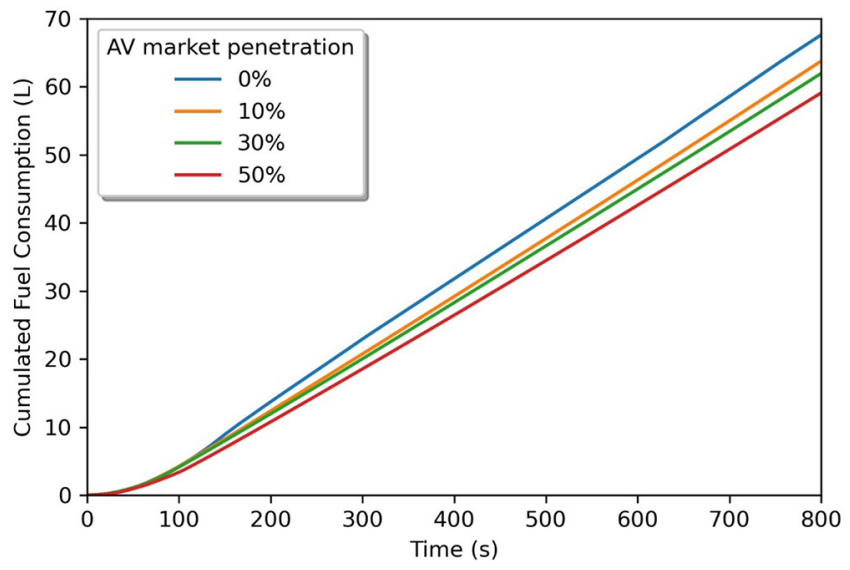

\section{Conclusions}

In this paper, we present a cooperative ACC, called SSDM, for CAVs in mixed traffic streams with human-driven vehicles. Considering the linear stability, SSDM is able to provide smooth deceleration and acceleration in the vehicle platoons with or without cut-in. Moreover, the calibrated VT-Micro is applied in this study to investigate the impact of CAVs on the fuel consumption of vehicle platoon and traffic flows.

Under the cut-in condition, all control strategies can reduce the oscillations caused by the cut-in vehicle when the desired time headway is $1.5 \mathrm{~s}$. When the desired time headway is $1 \mathrm{~s}$, the SDM is amplifying the oscillation while EcoSDM and SSDM are stable. Moreover, the SSDM outperforms EcoSDM and SDM in terms of stability considering different desired time headways.

Moreover, single-lane vehicle dynamics are simulated for human-driven vehicles and CAVs. The result shows that CAVs can reduce platoon-level fuel consumption. SSDM can save platoon-level fuel consumption up to $15 \%$ compared to human-driven vehicle platoons. SSDM outperforms the SDM and EcoSDM in terms of fuel consumption. Considering the single-lane highway with merging, the higher market penetration of SSDM-equipped CAVs leads to less fuel consumption.

The present paper has the following limitations. First, only one of the existing lateral maneuvers is considered in this study. Different combinations of longitudinal and lateral maneuvers should be applied to identify the best control strategy of CAVs. Moreover, field experiments will be conducted to evaluate the performance. Second, because human driving behavior may change because of the presence of autonomous driving technologies (Anesiadou et al., 2021; Pan et al., 2021; Shi et al., 2021; Zhao et al., 2020), the human driver behavior model, which is considered in the proposed strategy, needs to be updated according to the field data from mixed traffic in the future. Third, the performance of control strategies is evaluated based on freeway traffic conditions. In the future, network-level performance may be further evaluated. Moreover, the performance of the control methods based on machine learning, optimization and rules should be compared and discussed in future works. 


\section{Notes}

1 https://ruter.no/en/about-ruter/reports-projects-plans/autonomousvehicles/

2 https://www.epa.gov/vehicle-and-fuel-emissions-testing/ dynamometer-drive-schedules

3 https://www.epa.gov/vehicle-and-fuel-emissions-testing/ dynamometer-drive-schedules

\section{Reference}

Ahn, K., Rakha, H., Asce, M., Trani, A., Asce, M., Aerde, M. and Van Aerde, M. (2002), "Estimating vehicle fuel consumption and emissions based on instantaneous speed and acceleration levels", Fournal of Transportation Engineering, Vol. 128 No. 2, pp. 182-190.

Anesiadou, A., Makridis, M., Mattas, K., Fontaras, G. and Ciuffo, B. (2021), "Characterization of drivers heterogeneity and its integration within traffic simulation", ArXiv Preprint ArXiv:2107.02618, available at: http://arxiv.org/abs/2107.02618

Bando, M., Hasebe, K., Nakayama, A., Shibata, A. and Sugiyarna, Y. (1995), "Dynamical model of traffic congestion and numerical simulation", Physical Review E, Vol. 51 No. 2, pp. 1035-1042.

Chen, X., Li, R., Xie, W. and Shi, Q. (2009), "Stabilization of traffic flow based on multi-anticipative intelligent driver model", The 12th International IEEE Conference on Intelligent Transportation Systems, Proceedings, ITSC, St. Louis, pp. 72-77.

Chen, X.(M.)., Li, Z., Li, L. and Shi, Q. (2013), "Characterising scattering features in flow-density plots using a stochastic platoon model", Transportmetrica A: Transport Science, No. September, pp. 1-29.

Davis, L.C. (2004), "Effect of adaptive cruise control systems on traffic flow", Physical Review E, Vol. 69 No. 6, pp. 1-8.

Davis, L.C. (2007), "Effect of adaptive cruise control systems on mixed traffic flow near an on-ramp", Physica A: Statistical Mechanics and Its Applications, Vol. 379 No. 1, pp. 274-290.

Davis, L.C. (2017), "Dynamic origin-to-destination routing of wirelessly connected, autonomous vehicles on a congested network", Physica A: Statistical Mechanics and Its Applications, Vol. 478, pp. 93-102.

Desjardins, C. and Chaib-Draa, B. (2011), "Cooperative adaptive cruise control: a reinforcement learning approach", IEEE Transactions on Intelligent Transportation Systems, Vol. 12 No. 4, pp. 1248-1260.

Enang, W. and Bannister, C. (2017), "Modelling and control of hybrid electric vehicles (a comprehensive review)", Renewable and Sustainable Energy Reviews, Vol. 74 No. August 2016, pp. 1210-1239.

Fazi, M.B. (2020), "Beyond human: deep learning, explainability and representation", Theory, Culture and Society, doi: 10.1177/0263276420966386.

Gipps, P.G. (1981), “A behavioural car-following model for computer simulation", Transportation Research Part B: Methodological, Vol. 15 No. 2, pp. 105-111.

Gunter, G., Gloudemans, D., Stern, R.E., McQuade, S., Bhadani, R., Bunting, M., Delle Monache, M.L., et al. (2019), "Are commercially implemented adaptive cruise control systems string stable?", Ieee Transactions on Intelligent Transportation Systems, pp. 1-12.

Gunter, G., Janssen, C., Barbour, W., Stern, R.E. and Work, D.B. (2020), "Model based string stability of adaptive cruise control systems using field data", IEEE Transactions on Intelligent Vehicles, Vol. 5 No. 1, pp. 90-99.

He, D., He, W. and Song, X. (2020), "Efficient predictive cruise control of autonomous vehicles with improving ride comfort and safety", Measurement and Control, Vol. 53 Nos 1/2, pp. 18-28.

Huang, K., Yang, X., Lu, Y., Mi, C.C. and Kondlapudi, P. (2018), "Ecological driving system for connected/automated vehicles using a two-stage control hierarchy", IEEE Transactions on Intelligent Transportation Systems, Vol. 19 No. 7, pp. 2373-2384.

Huang, L., Zhai, C., Wang, H., Zhang, R., Qiu, Z. and Wu, J. (2020), "Cooperative adaptive cruise control and exhaust emission evaluation under heterogeneous connected vehicle network environment in urban city", Fournal of Environmental Management, Vol. 256 No. September 2018, p. 109975.

Ioannou, P.A. and Stefanovic, M. (2005), "Evaluation of ACC vehicles in mixed traffic: lane change effects and sensitivity analysis", IEEE Transactions on Intelligent Transportation Systems, Vol. 6 No. 1, pp. 79-89.

Jiang, R., Wu, Q. and Zhu, Z. (2001), "Full velocity difference model for a car-following theory", Physical Review E, Vol. 64 No. 1, p. 017101.

Jiao, S., Zhang, S., Zhou, B., Zhang, L. and Xue, L. (2021), "Dynamic performance and safety analysis of car-following models considering collision sensitivity", Physica A: Statistical Mechanics and Its Applications, Elsevier B.V., Vol. 564, p. 125504.

Kamal, M.A.S., Mukai, M., Murata, J. and Kawabe, T. (2011), "Ecological vehicle control on roads with up-down slopes", IEEE Transactions on Intelligent Transportation Systems, Vol. 12 No. 3, pp. 783-794.

Kazemi, H., Mahjoub, H.N., Tahmasbi-Sarvestani, A. and Fallah, Y.P. (2018), "A learning-based stochastic MPC design for cooperative adaptive cruise control to handle interfering vehicles", IEEE Transactions on Intelligent Vehicles, Vol. 3 No. 3, pp. 266-275.

Kesting, A., Treiber, M. and Helbing, D. (2010), "Enhanced intelligent driver model to access the impact of driving strategies on traffic capacity", Philosophical Transactions of the Royal Society A: Mathematical, Physical and Engineering Sciences, Vol. 368 No. 1928, pp. 4585-4605.

Kesting, A., Treiber, M., Schönhof, M. and Helbing, D. (2008), "Adaptive cruise control design for active congestion avoidance", Transportation Research Part C: Emerging Technologies, Vol. 16 No. 6, pp. 668-683.

Kuutti, S., Bowden, R., Jin, Y., Barber, P. and Fallah, S. (2021), "A survey of deep learning applications to autonomous vehicle control", IEEE Transactions on Intelligent Transportation Systems, Vol. 22 No. 2, pp. 712-733.

Lenz, H., Wagner, C.K. and Sollacher, R. (1999), "Multianticipative car-following model”, The European Physical Fournal B, Vol. 7 No. 2, pp. 331-335.

Li, G. and Görges, D. (2020), "Ecological adaptive cruise control for vehicles with Step-Gear transmission based on 
reinforcement learning", IEEE Transactions on Intelligent Transportation Systems, Vol. 21 No. 11, pp. 4895-4905.

Li, S.E., Jia, Z., Li, K. and Cheng, B. (2015a), "Fast online computation of a model predictive controller and its application to fuel economy oriented adaptive cruise control”, IEEE Transactions on Intelligent Transportation Systems, Vol. 16 No. 3, pp. 1199-1209.

Li, Z., Li, W., Xu, S. and Qian, Y. (2015b), "Stability analysis of an extended intelligent driver model and its simulations under open boundary condition", Physica A: Statistical Mechanics and Its Applications, Vol. 419, pp. 526-536.

Lin, Y., McPhee, J. and Azad, N.L. (2021), "Comparison of deep reinforcement learning and model predictive control for adaptive cruise control", IEEE Transactions on Intelligent Vehicles, Vol. 6 No. 2, pp. 221-231.

Lu, C. and Aakre, A. (2018), "A new adaptive cruise control strategy and its stabilization effect on traffic flow", European Transport Research Review, Vol. 10 No. 2, doi: 10.1186/ s12544-018-0321-9.

Lu, C., Dong, J. and Hu, L. (2019a), "Energy-Efficient adaptive cruise control for electric connected and autonomous vehicles", IEEE Intelligent Transportation Systems Magazine, Vol. 11 No. 3, pp. 42-55.

Lu, C., Dong, J., Hu, L. and Liu, C. (2019b), "An ecological adaptive cruise control for mixed traffic and its stabilization effect", IEEE Access, Vol. 7, pp. 81246-81256.

Lu, C. Hu, L. and Dong, J. (2018), "Ecological adaptive cruise control in a traffic stream with mixed autonomous and manually driven vehicles".

Luo, Y., Chen, T., Zhang, S. and Li, K. (2015), "Intelligent hybrid electric vehicle ACC with coordinated control of tracking ability, fuel economy, and ride comfort", IEEE Transactions on Intelligent Transportation Systems, Vol. 16 No. 4.

Mahdinia, I., Arvin, R., Khattak, A.J. and Ghiasi, A. (2020), "Safety, energy, and emissions impacts of adaptive cruise control and cooperative adaptive cruise control", Transportation Research Record: Fournal of the Transportation Research Board, Vol. 2674 No. 6, pp. 253-267.

Makridis, M., Mattas, K., Ciuffo, B., Re, F., Kriston, A., Minarini, F. and Rognelund, G. (2020), "Empirical study on the properties of adaptive cruise control systems and their impact on traffic flow and string stability", Transportation Research Record: Fournal of the Transportation Research Board, Vol. 2674 No. 4, pp. 471-484.

Milanés, V. and Shladover, S.E. (2016), "Handling cut-in vehicles in strings of cooperative adaptive cruise control vehicles", fournal of Intelligent Transportation Systems, Vol. 20 No. 2, pp. 178-191.

Newell, G.F.F. (1961), "Nonlinear effects in the dynamics of car Following", Operations Research, Vol. 9 No. 2, pp. 209-229.

Nunen, E., Van, Reinders, J., Semsar-Kazerooni, E. Wouw, N. and Van De, (2019), "String stable model predictive cooperative adaptive cruise control for heterogeneous platoons", IEEE Transactions on Intelligent Vehicles, Vol. 4 No. 2, pp. 186-196.

Pan, C., Xu, J. and Fu, J. (2021), "Effect of gender and personality characteristics on the speed tendency based on advanced driving assistance system (ADAS) evaluation", Fournal of Intelligent and Connected Vehicles, pp. 28-37.

Pei, X., Pan, Y., Wang, H., Wong, S.C. and Choi, K. (2016), "Empirical evidence and stability analysis of the linear carfollowing model with gamma-distributed memory effect", Physica A: Statistical Mechanics and Its Applications, Vol. 449, pp. 311-323.

Rakha, H. and Ahn, K. (2004), "Integration modeling framework for estimating mobile source emissions", fournal of Transportation Engineering, Vol. 130 No. 2, pp. 183-193.

Sakhdari, B. and Azad, N.L. (2018), "Adaptive tube-based nonlinear MPC for ecological autonomous cruise control of plug-in hybrid electric vehicles", IEEE Transactions on Vehicular Technology, Vol. 67 No. 12, pp. 11390-11401.

Shang, M. and Stern, R.E. (2021), "Impacts of commercially available adaptive cruise control vehicles on highway stability and throughput", Transportation Research Part C: Emerging Technologies, Vol. 122 No. June 2020, p. 102897.

Shi, X., Wang, Z., Li, X. and Pei, M. (2021), "The effect of ride experience on changing opinions toward autonomous vehicle safety", Communications in Transportation Research, Vol. 1 No. July, p. 100003.

Sun, D., Chen, D., Zhao, M., Liu, W. and Zheng, L. (2018), "Linear stability and nonlinear analyses of traffic waves for the general nonlinear car-following model with multi-time delays", Physica A: Statistical Mechanics and Its Applications, Vol. 501, pp. 293-307.

Tajeddin, S., Ekhtiari, S., Faieghi, M. and Azad, N.L. (2020), "Ecological adaptive cruise control with optimal lane selection in connected vehicle environments", IEEE Transactions on Intelligent Transportation Systems, Vol. 21 No. 11, pp. 4538-4549.

Talebpour, A. and Mahmassani, H.S. (2016), "Influence of connected and autonomous vehicles on traffic flow stability and throughput", Transportation Research Part C: Emerging Technologies, Vol. 71 No. October, pp. 143-163.

Tampere, C.M.J. (2004), "Human-Kinetic multiclass traffic flow theory and modelling with application to advanced driver assistance systems in congestion", Thesis.

Wang, C., Gong, S., Zhou, A., Li, T. and Peeta, S. (2020), "Cooperative adaptive cruise control for connected autonomous vehicles by factoring communication-related constraints", Transportation Research Part C: Emerging Technologies, Vol. 113 No. March 2019, pp. 124-145.

Wang, M., Hoogendoorn, S.P., Daamen, W., van Arem, B., Shyrokau, B. and Happee, R. (2018), "Delay-compensating strategy to enhance string stability of autonomous vehicle platoons", Transportmetrica B: Transport Dynamics, Vol. 6 No. 3, pp. 211-229.

Wilson, R.E. and Ward, J.A. (2011), "Car-following models: fifty years of linear stability analysis - a mathematical perspective", Transportation Planning and Technology, Vol. 34 No. 1, pp. 3-18.

Xue, W., Zheng, R., Yang, B., Wang, Z., Kaizuka, T. and Nakano, K. (2019), “An adaptive model predictive approach for automated vehicle control in fallback procedure based on virtual vehicle scheme", fournal of Intelligent and Connected Vehicles, Vol. 2 No. 2, pp. 67-77.

Yang, Y., Ma, F., Wang, J., Zhu, S., Gelbal, S.Y., KavasTorris, O., Aksun-Guvenc, B., et al. (2020), "Cooperative 
ecological cruising using hierarchical control strategy with optimal sustainable performance for connected automated vehicles on varying road conditions", fournal of Cleaner Production, Vol. 275, p. 123056.

Yuan, Y., Jiang, R., Hu, M., Wu, Q. and Wang, R. (2009), "Traffic flow characteristics in a mixed traffic system consisting of ACC vehicles and manual vehicles: a hybrid modelling approach", Physica A: Statistical Mechanics and Its Applications, Vol. 388 No. 12, pp. 2483-2491.

Zhao, X., Wang, Z., Xu, Z., Wang, Y., Li, X. and Qu, X. (2020), "Field experiments on longitudinal characteristics of human driver behavior following an autonomous vehicle",
Transportation Research Part C: Emerging Technologies, Vol. 114 No. February, pp. 205-224.

Zheng, Z. (2021), "Reasons, challenges, and some tools for doing reproducible transportation research", Communications in Transportation Research, Vol. 1 No. July, p. 100004.

Zhu, Y., He, H. and Zhao, D. (2020), "LMI-Based synthesis of String-Stable controller for cooperative adaptive cruise control", IEEE Transactions on Intelligent Transportation Systems, Vol. 21 No. 11, pp. 4516-4525.

\section{Corresponding author}

Chaoru Lu can be contacted at: chaorulu@oslomet.no

For instructions on how to order reprints of this article, please visit our website: 